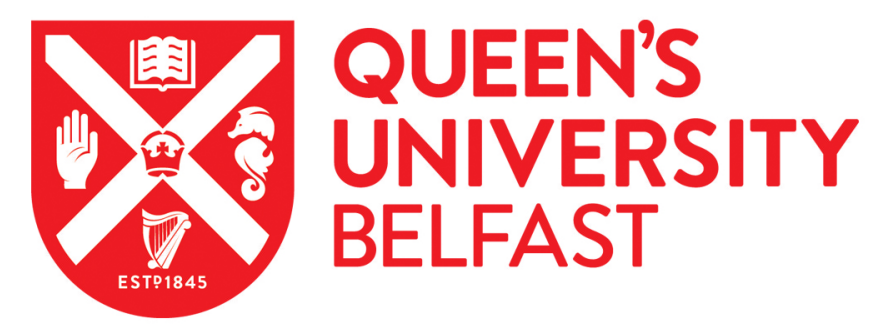

\title{
Positronium-atom scattering at low energies
}

Fabrikant, I. I., \& Gribakin, G. F. (2014). Positronium-atom scattering at low energies. Physical Review A (Atomic, Molecular, and Optical Physics), 90, 052717. [052717]. https://doi.org/10.1103/PhysRevA.90.052717

Published in:

Physical Review A (Atomic, Molecular, and Optical Physics)

Document Version:

Peer reviewed version

Queen's University Belfast - Research Portal:

Link to publication record in Queen's University Belfast Research Portal

Publisher rights

(C) 2014 American Physical Society

\section{General rights}

Copyright for the publications made accessible via the Queen's University Belfast Research Portal is retained by the author(s) and / or other copyright owners and it is a condition of accessing these publications that users recognise and abide by the legal requirements associated with these rights.

Take down policy

The Research Portal is Queen's institutional repository that provides access to Queen's research output. Every effort has been made to ensure that content in the Research Portal does not infringe any person's rights, or applicable UK laws. If you discover content in the Research Portal that you believe breaches copyright or violates any law, please contact openaccess@qub.ac.uk. 


\title{
Ps-atom scattering at low energies
}

\author{
I. I. Fabrikant \\ Department of Physics and Astronomy, \\ University of Nebraska, Lincoln, Nebraska 68588-0299, USA \\ G. F. Gribakin \\ School of Mathematics and Physics, Queen's University Belfast, \\ Belfast BTr 1NN, Northern Ireland, United Kingdom
}

(Dated: November 17, 2014)

\begin{abstract}
A pseudopotential for positronium-atom interaction, based on electron-atom and positron-atom phase shifts, is constructed, and the phase shifts for Ps-Kr and Ps-Ar scattering are calculated. This approach allows us to extend the Ps-atom cross sections, obtained previously in the impulse approximation [Phys. Rev. Lett. 112, 243201 (2014)], to energies below the Ps ionization threshold. Although experimental data are not available in this low-energy region, our results describe well the tendency of the measured cross sections to drop with decreasing velocity at $v<1$ a.u. Our results show that the effect of the Ps-atom van der Waals interaction is weak compared to the polarization interaction in electron-atom and positron-atom scattering. As a result, the Ps scattering length for both $\mathrm{Ar}$ and $\mathrm{Kr}$ is positive, and the Ramsauer-Townsend minimum is not observed for Ps scattering from these targets. This makes Ps scattering quite different from electron scattering in the low-energy region, in contrast to the intermediate energy range from the Ps ionization threshold up to $v \sim 2$ a.u., where the two are similar.
\end{abstract}

PACS numbers: 34.80.-i, 36.10.Dr 


\section{INTRODUCTION}

Recently observed similarities between the positronium scattering and the electron scattering from a number of atoms and molecules [1-3] in the intermediate energy range suggest that both processes are largely controlled by the same interactions. When plotted as a function of the projectile velocity, the electron and Ps cross sections are very close and even show similar resonancelike features. This similarity was explained recently [4] using the impulse approximation. It was shown that above the Ps ionization threshold, the Ps- $A$ interaction is mainly controlled by the $e^{-}-A$ scattering amplitude, and the $e^{-}-A$ exchange contributes mostly to this amplitude in the intermediate energy range. It is clear, however, that at higher energies, the role of the exchange interaction becomes less significant, and the similarity between Ps- $A$ and $e^{-}-A$ scattering should gradually disappear. On the other hand, at lower energies, long-range interactions between the projectile and the target play a significant role, and they are substantially different for $e^{-}-A$ and Ps- $A$ interactions. In the former case it is the polarization potential decreasing as $r^{-4}$ at large distances $r$, and in the latter case the van der Waals interaction decreasing as $r^{-6}$.

The impulse approximation for Ps- $A$ scattering [4] produces very large cross sections below the ionization threshold because of the dominance of the $e^{+}-A$ scattering amplitude. This growth of the Ps cross section is unphysical, since the large $e^{+}-A$ amplitude is due to the effects of positron-atom polarization and virtual Ps formation, both of which are absent in Ps- $A$ scattering. Since the impulse approximation breaks down at energies below the ionization threshold, alternative methods and approximations, such as close-coupling and static-exchange, should be used in this energy range. Blackwood et al. [5] performed closecoupling calculations of Ps scattering from noble-gas atoms. These calculations allowed for the distortion and break-up of Ps, but kept the target "frozen", i.e., they neglected any excitations of the target. Virtual target excitations are known to be very important in lowenergy electron- and positron-atom collisions, where they can be described in terms of the polarization interaction. For low-energy Ps-atom collisions, they give rise to the van der Waals interaction, which can be incorporated by extending the close-coupling calculations to include the virtual excitations of the target. Such calculations have been performed for Ps collisions with the hydrogen atom $[6,7]$ and would be an ultimate goal in the problem of Ps-atom collisions. However, they are very challenging computationally for complex atoms. 
In this paper we develop an alternative low-energy method based on the use of the electron and positron scattering phase shifts, similar to the impulse approximation. It involves constructing model potentials that reproduce these phase shifts, and then adding them to describe the Ps-atom interaction. While this procedure is straightforward for positron scattering, the situation with electrons is more complicated. Due to the Pauli exclusion principle, the effective potential for the electron depends on its orbital angular momentum $l$, i.e., it becomes a pseudopotential [8]. When such a pseudopotential is averaged over the electron density distribution in the Ps atom, it becomes a nonlocal operator.

Another difficulty is related to inclusion of the long-range interaction. Accurate lowenergy electron and positron scattering phase shifts contain contributions of the atomic polarization potential. This potential is attractive for both electrons and positrons and behaves as $-\alpha / 2 r^{4}$ at large distances, where $\alpha$ is the atomic dipole polarizability. (We use atomic units throughout.) An effective Ps- $A$ potential including such contributions would behave as $-\alpha / r^{4}$, which is physically incorrect, as the dominant long-range Ps- $A$ interaction is the van der Waals interaction $-C_{6} / R^{6}$. The latter potential results from the manybody Ps-atom, rather than single-particle (i.e., electron-atom or positron-atom) dynamics. It can be obtained by including the two-body polarization potential (see, e.g., [9]) in the Hamiltonian, which gives the total polarization interaction at large distances as

$$
V_{\mathrm{pol}}\left(\mathbf{r}_{e}, \mathbf{r}_{p}\right)=-\frac{\alpha}{2 r_{e}^{4}}-\frac{\alpha}{2 r_{p}^{4}}+\frac{\alpha \mathbf{r}_{e} \cdot \mathbf{r}_{p}}{r_{e}^{3} r_{p}^{3}}
$$

where $\mathbf{r}_{e}$ and $\mathbf{r}_{p}$ are the electron and positron position vectors, respectively, relative to the target. Averaging of this potential over the electron and positron density distribution in Ps does lead to an effective van der Waals interaction [10].

Alternatively, one can construct the positron-atom and electron-atom pseudopotentials using static (static-exchange) phase shifts for the positron (electron), i.e., neglecting the polarization. The Ps-atom van der Waals interaction can then be added, e.g., in the form

$$
V_{W}(R)=-\frac{C_{6}}{R^{6}}\left\{1-\exp \left[-\left(R / R_{c}\right)^{8}\right]\right\}
$$

where $C_{6}$ is the van der Waals constant and $R_{c}$ is a cutoff radius. The $C_{6}$ values for Psatom pairs are known, e.g., from the London formula [11], which gives $C_{6}=152$ a.u. for Kr and $C_{6}=104.5$ a.u. for Ar. (These values are close to the estimates obtained in Ref. [12].) In contrast, the cutoff parameter $R_{c}$ cannot be determined rigorously, but the phase 
shifts and cross sections are sensitive to its choice. A similar problem is encountered when using the polarization interaction (1), which also requires a cut-off at small distances. In the present calculations the radius $R_{c}$ is set by requiring that the cross sections given by the pseudopotential method merge smoothly with the elastic cross section calculated in the impulse approximation above the Ps ionization threshold [4].

The rest of the article is organized as follows. First we discuss the construction of the pseudopotentials for $e^{+}$and $e^{-}$scattering from the static (static-exchange) phase shifts, and the derivation of the pseudopotential for Ps-atom scattering. We then present the results for Ps-Kr and Ps-Ar scattering and discuss the low-energy behavior of the cross sections. For both atoms, the scattering length is positive, which implies effective repulsion at low energies and rules out the existence of the Ramsauer-Townsend minimum.

\section{THEORY}

\section{A. Pseudopotentials}

We choose the positron-atom pseudopotential in the form

$$
V_{p}(r)=\frac{Z_{p}}{r} e^{-\alpha_{p} r}
$$

which represents the static $e^{+}-A$ repulsion, and where $Z_{p}$ and $\alpha_{p}$ are fitting parameters. They are obtained by fitting the $s^{-}, p$ - and $d$-wave scattering phase shifts in the potential (3) to the positron scattering phase shifts in the static field of the ground-state atom calculated in the Hartee-Fock approximation (see Sec. IIB). The parameter $Z_{p}$ plays the role of an effective nuclear charge. It can be different from the actual nuclear charge, since a low-energy positron does not penetrate deep into the atom.

The effective static-exchange potential for the electron is chosen as

$$
V_{e}(r)=-\frac{Z_{e}}{r} e^{-\alpha_{e} r}+\frac{B}{r^{n}} e^{-\beta r}
$$

where the second term represents repulsion due to the Pauli exclusion principle. This effect depends on the orbitals occupied in the atomic ground state, hence it is $l$-dependent. We also found that in general the ab initio static-exchange (Hartree-Fock) phase shifts cannot be reproduced using $Z_{e}=Z_{p}$. Therefore, we regard all parameters in Eq. (4) as l-dependent. 
Formally this means that the effective electron-atom potential is a nonlocal operator with the kernel

$$
V_{e}\left(\mathbf{r}, \mathbf{r}^{\prime}\right)=\frac{1}{r^{2}} \delta\left(r-r^{\prime}\right) \sum_{l m} V_{l}(r) Y_{l m}^{*}(\hat{\mathbf{r}}) Y_{l m}\left(\hat{\mathbf{r}}^{\prime}\right)
$$

where $V_{l}(r)$ are potentials given by Eq. (4). It is convenient to rewrite this expression as

$$
V_{e}\left(\mathbf{r}, \mathbf{r}^{\prime}\right)=-V_{p}(r) \delta\left(\mathbf{r}-\mathbf{r}^{\prime}\right)+\frac{1}{r^{2}} \delta\left(r-r^{\prime}\right) \sum_{l m} v_{l}(r) Y_{l m}^{*}(\hat{\mathbf{r}}) Y_{l m}\left(\hat{\mathbf{r}}^{\prime}\right)
$$

where

$$
v_{l}(r)=V_{l}(r)+V_{p}(r)
$$

Since the "direct" part of the potential $V_{l}(r)$ is close in magnitude, but opposite in sign to $V_{p}(r), v_{l}(r)$ represents mainly the exchange interaction between the electron and the atom.

The Ps-atom pseudopotential can be now written as

$$
V_{\mathrm{Ps}}\left(\mathbf{r}_{e}, \mathbf{r}_{e}^{\prime}, \mathbf{r}_{p}, \mathbf{r}_{p}^{\prime}\right)=V_{p}\left(r_{p}\right) \delta\left(\mathbf{r}_{p}-\mathbf{r}_{p}^{\prime}\right)-V_{p}\left(r_{e}\right) \delta\left(\mathbf{r}_{e}-\mathbf{r}_{e}^{\prime}\right)+\frac{1}{r_{e}^{2}} \delta\left(r_{e}-r_{e}^{\prime}\right) \sum_{l m} v_{l}\left(r_{e}\right) Y_{l m}^{*}\left(\hat{\mathbf{r}}_{e}\right) Y_{l m}\left(\hat{\mathbf{r}}_{e}^{\prime}\right) .
$$

In the static approximation we average this potential over the electron and positron density distribution in Ps given by $|\Phi(\boldsymbol{\rho})|^{2}$, where $\Phi(\boldsymbol{\rho})$ is the Ps ground-state wave function and $\boldsymbol{\rho}$ is the relative $e^{-}-e^{+}$coordinate. The relations between $\mathbf{r}_{e}, \mathbf{r}_{p}$ and $\boldsymbol{\rho}$ are

$$
\mathbf{r}_{e}=\mathbf{R}+\boldsymbol{\rho} / 2, \quad \mathbf{r}_{p}=\mathbf{R}-\boldsymbol{\rho} / 2
$$

where $\mathbf{R}$ is the position of the Ps center of mass relative to the target.

The average of the local part of the pseudopotential (8) reduces to the integral

$$
\int\left[V_{p}(\mathbf{R}-\boldsymbol{\rho} / 2)-V_{p}(\mathbf{R}+\boldsymbol{\rho} / 2)\right]|\Phi(\boldsymbol{\rho})|^{2} d \boldsymbol{\rho},
$$

which vanishes because the integrand is parity odd. This corresponds to a well-known fact that the static potential for the Ps- $A$ interaction is zero. The remaining nonlocal part in Eq. (8) contains a strong repulsive core, and to make the calculations more tractable, it is convenient to represent $v_{l}\left(r_{e}\right)$ as

$$
v_{l}\left(r_{e}\right)=v_{\mathrm{loc}}\left(r_{e}\right)+u_{l}\left(r_{e}\right),
$$

where the $l$-independent part $v_{\text {loc }}\left(r_{e}\right)$ contains the major repulsive contribution, and $u_{l}\left(r_{e}\right)$ accounts for the remaining $l$-dependent part. The averaging procedure is then reduced to 
averaging of the Ps pseudopotential

$$
\tilde{V}_{\mathrm{Ps}}\left(\mathbf{r}_{e}, \mathbf{r}_{e}^{\prime}\right)=v_{\mathrm{loc}}\left(r_{e}\right) \delta\left(\mathbf{r}_{e}-\mathbf{r}_{e}^{\prime}\right)+\frac{1}{r_{e}^{2}} \delta\left(r_{e}-r_{e}^{\prime}\right) \sum_{l m} u_{l}\left(r_{e}\right) Y_{l m}^{*}\left(\hat{\mathbf{r}}_{e}\right) Y_{l m}\left(\hat{\mathbf{r}}_{e}^{\prime}\right) .
$$

The average of the local part of this potential gives the local Ps-atom potential

$$
v_{\mathrm{av}}(R)=\int v_{\mathrm{loc}}(\mathbf{R}+\boldsymbol{\rho} / 2)|\Phi(\boldsymbol{\rho})|^{2} d \boldsymbol{\rho},
$$

while averaging the nonlocal part [second term in Eq. (10)] gives a nonlocal contribution to the $\mathrm{Ps}_{\mathrm{S}} A$ interaction,

$$
V\left(\mathbf{R}, \mathbf{R}^{\prime}\right)=\sum_{l m} \int \frac{1}{r^{2}} \delta\left(r-r^{\prime}\right) u_{l}(r) Y_{l m}^{*}(\hat{\mathbf{r}}) Y_{l m}\left(\hat{\mathbf{r}}^{\prime}\right)|\Phi(\boldsymbol{\rho})|^{2} d \boldsymbol{\rho}
$$

where $\mathbf{r}=\mathbf{R}+\boldsymbol{\rho} / 2$ and $\mathbf{r}^{\prime}=\mathbf{R}^{\prime}+\boldsymbol{\rho} / 2$.

In performing these integrations, it is more convenient to switch to the integration variable $\mathbf{r}$. Since the result depends only on the absolute magnitude of the vectors $\mathbf{R}$ and $\mathbf{R}^{\prime}$, and on the angle between them, the integration in Eq. (12) can be performed in the coordinate system with the polar axis along the vector

$$
\mathbf{s}=\mathbf{R}^{\prime}-\mathbf{R}
$$

We then have

$$
V\left(\mathbf{R}, \mathbf{R}^{\prime}\right)=8 \sum_{l} \frac{2 l+1}{4 \pi} \int \frac{1}{r^{2}} \delta\left(r-r^{\prime}\right) u_{l}(r) P_{l}\left(\cos \theta_{\mathbf{r r}^{\prime}}\right)|\Phi(2(\mathbf{r}-\mathbf{R}))|^{2} d \mathbf{r},
$$

where $\mathbf{r}^{\prime}=\mathbf{r}+\mathbf{s}$ and $\theta_{\mathbf{r} \mathbf{r}^{\prime}}$ is the angle between $\mathbf{r}$ and $\mathbf{r}^{\prime}$.

Integration over the polar angle $\theta$ in this coordinate system eliminates the $\delta$-function (which ensures $r=r^{\prime}$ ) and fixes the angles:

$$
\cos \theta=-\frac{s}{2 r}, \quad \cos \theta_{\mathbf{r r}^{\prime}}=\frac{r+s \cos \theta}{|\mathbf{r}+\mathbf{s}|}=1-\frac{s^{2}}{2 r^{2}} .
$$

It also introduces a factor $1 / s$ since

$$
\frac{d(r-|\mathbf{r}+\mathbf{s}|)}{d \cos \theta}=-s
$$

for $\cos \theta=-s / 2 r$.

The Ps ground-state density is expanded in spherical harmonics as

$$
|\Phi(2|\mathbf{r}-\mathbf{R}|)|^{2}=\frac{1}{8 \pi} e^{-2|\mathbf{r}-\mathbf{R}|}=\frac{1}{8 \pi} \sum_{l^{\prime}=0}^{\infty} F_{l^{\prime}}(r, R)\left(2 l^{\prime}+1\right) P_{l^{\prime}}\left(\cos \theta_{\mathbf{r R}}\right),
$$


where the expression for $F_{l}(r, R)$ is given in Appendix A (see also Appendix B in Ref. [13]).

The Legendre polynomial $P_{l^{\prime}}\left(\cos \theta_{\mathbf{r R}}\right)$ is the only part of expansion (14) which depends on the azimuthal angle $\phi$ in the integrand of Eq. (13) $\left(\theta_{\mathbf{r r}^{\prime}}\right.$ does not depend on $\left.\phi\right)$. Therefore we can perform integration over $\phi$ as

$$
\int_{0}^{2 \pi} P_{l^{\prime}}\left(\cos \theta_{\mathbf{r R}}\right) d \phi=2 \pi P_{l^{\prime}}(\cos \theta) P_{l^{\prime}}\left(\cos \theta_{R}\right)
$$

where

$$
\cos \theta_{R}=\frac{R^{\prime} \cos \Theta-R}{s}
$$

and $\Theta$ is the angle between $\mathbf{R}$ and $\mathbf{R}^{\prime}$.

Thus, we obtain the nonlocal part of the Ps-atom potential as

$V\left(\mathbf{R}, \mathbf{R}^{\prime}\right)=\frac{1}{2 \pi s} \sum_{l l^{\prime}}(2 l+1)\left(2 l^{\prime}+1\right) P_{l^{\prime}}\left(\cos \theta_{R}\right) \int_{0}^{\infty} P_{l}\left(1-\frac{s^{2}}{2 r^{2}}\right) P_{l^{\prime}}\left(-\frac{s}{2 r}\right) F_{l^{\prime}}(r, R) u_{l}(r) d r$.

It is convenient to expand this expression in Legendre polynomials,

$$
V\left(\mathbf{R}, \mathbf{R}^{\prime}\right)=\frac{1}{R R^{\prime}} \sum_{L=0}^{\infty} \frac{2 L+1}{4 \pi} V_{L}\left(R, R^{\prime}\right) P_{L}(\cos \Theta),
$$

where

$$
V_{L}\left(R, R^{\prime}\right)=2 \pi R R^{\prime} \int_{0}^{\pi} V\left(\mathbf{R}, \mathbf{R}^{\prime}\right) P_{L}(\cos \Theta) \sin \Theta d \Theta .
$$

Similarly, for the local part of the interaction potential, given by Eq. (11), we obtain

$$
v_{\mathrm{av}}(R)=4 \int_{0}^{\infty} v_{\mathrm{loc}}(r) F_{0}(r, R) r^{2} d r
$$

Substitution of the local and nonlocal potentials in the Schrödinger equation for the Ps- $A$ system yields a set of radial equations:

$$
\frac{1}{2 m} \frac{d^{2} f_{L}}{d R^{2}}+\left[E-v_{a v}(R)-\frac{L(L+1)}{2 m R^{2}}\right] f_{L}(R)-\int V_{L}\left(R, R^{\prime}\right) f_{L}\left(R^{\prime}\right) d R^{\prime}=0,
$$

where $m=2$ a.u. is the Ps mass, and $f_{L}(R)$ is the radial part of the Ps center-of-mass wave function for the orbital angular momentum $L$.

The sums in Eq. (15) converge fast. With a proper choice of $v_{\text {loc }}\left(r_{e}\right)$, the sum over $l$ can be truncated at $l_{\max }=2$. Increasing $l_{\max }$ to 4 has almost no effect on the phase shifts in the velocity range up to 2 a.u. The sum over $l^{\prime}$ converges if $l_{\max }^{\prime} \geq 6$. 


\section{B. Fitting parameters}

The values of the parameters of the pseudopotentials in Eqs. (3) and (4), were determined by fitting the positron-atom and electron-atom scattering phase shifts obtained in the static potential of the atom calculated in the Hartree-Fock approximation [14].

As mentioned in Sec. II A, for the positron, the same pseudopotential can be used for all partial waves. The static-field scattering phase shifts for the positron on Ar are shown in Fig. 1, and the corresponding sets of parameters for $\mathrm{Ar}$ and $\mathrm{Kr}$ are given in Table I. For positron velocities up to 2 a.u., the pseudopotential phase shifts are within $1 \%$ of the actual static-field phase shifts and are indistinguishable from them on the scale of the plot.

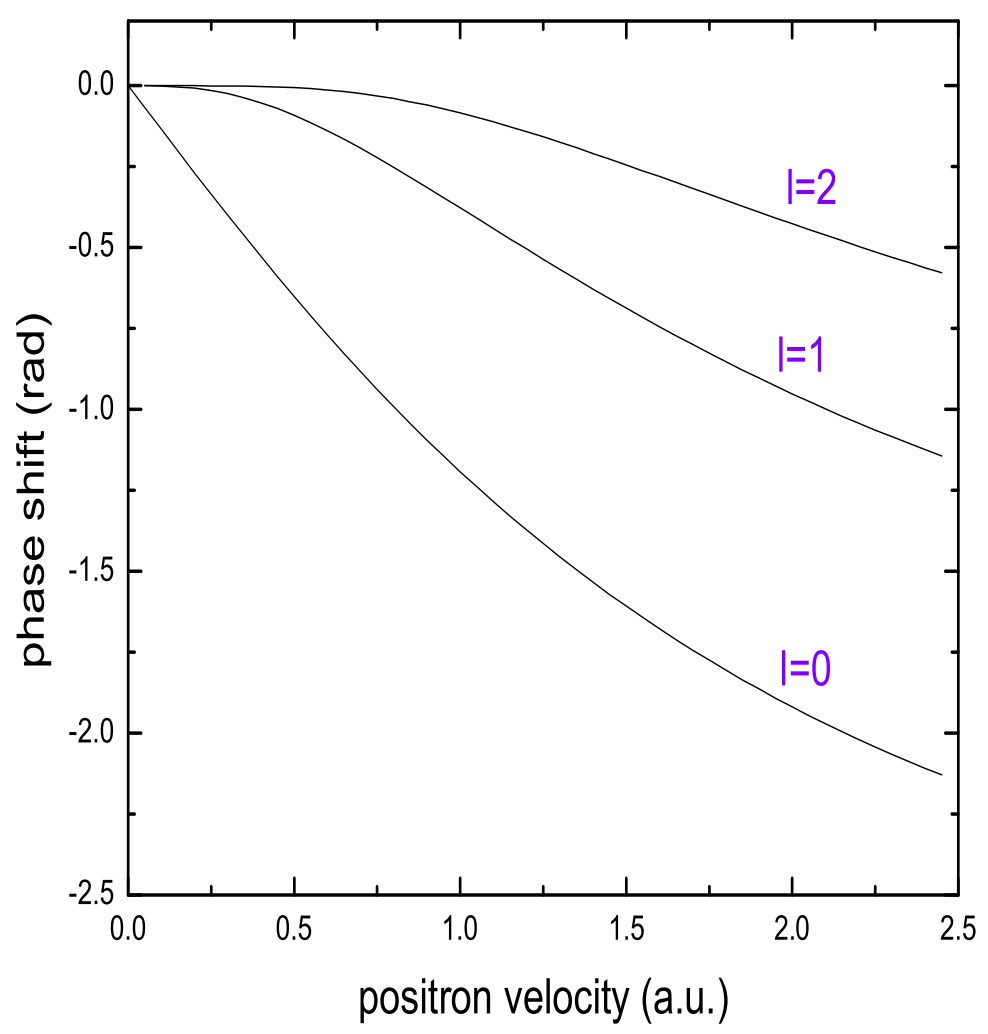

FIG. 1: Positron scattering phase shifts for Ar obtained using the static potential of the HartreeFock atom.

For the electron, the pseudopotential is optimized separately for each partial wave, by fitting the Hartree-Fock scattering phase shifts. The corresponding sets of parameters for Ar and $\mathrm{Kr}$ are given in Table I, and the phase shifts for Ar are shown in Fig. 2 for $l=0-2$. The pseudopotential for the $s$ wave gives the phase shifts that are almost indistinguishable from 
TABLE I: Parameters of the positron and electron pseudopotentials, given by Eqs. (3) and (4), for Ar and Kr.

\begin{tabular}{ccccccc}
\hline \hline System & $l$ & $Z$ & $\alpha_{p, e}$ & $B$ & $n$ & $\beta$ \\
\hline$e^{+}-\mathrm{Ar}$ & $0-4$ & 18.06 & 1.95 & - & - & - \\
\hline$e^{-}-\mathrm{Ar}$ & 0 & 4.297 & 0.618 & 14.72 & 3 & 0.248 \\
& 1 & 10.0 & 2.368 & 15.24 & 3 & 0.504 \\
& 2 & 9.780 & 1.230 & 0 & - & - \\
& 3 & 12.48 & 1.544 & 0 & - & - \\
& 4 & 15.13 & 1.714 & 0 & - & - \\
\hline$e^{+}-\mathrm{Kr}$ & $0-4$ & 20.79 & 1.760 & - & - & - \\
\hline$e^{-}-\mathrm{Kr}$ & 0 & 20.79 & 1.760 & 56.84 & 6 & 0 \\
& 1 & 20.79 & 1.760 & 97.80 & 6 & - \\
& 2 & 18.25 & 1.317 & 0 & - & - \\
& 3 & 14.80 & 1.409 & 0 & - & - \\
\hline \hline
\end{tabular}

the Hartree-Fock, and the fitted phase shifts for the $p$ and $d$ waves are also quite accurate. The behavior of the positron and electron phase shifts and the quality of the pseudopotential fits for Kr are similar to those shown for Ar. For both atoms, the $d$-wave displays a broad resonance at the electron velocity $v \sim 1$ a.u. (see Fig. 2).

\section{RESULTS AND DISCUSSION}

In this section, we present the Ps-atom scattering phase shifts calculated from Eq. (16) which contains the static Ps pseudopotential, and with the inclusion of the van der Waals interaction (2). We also use these phase shifts to compute the Ps-atom elastic scattering cross section, and compare our results with experiment and other theories. 


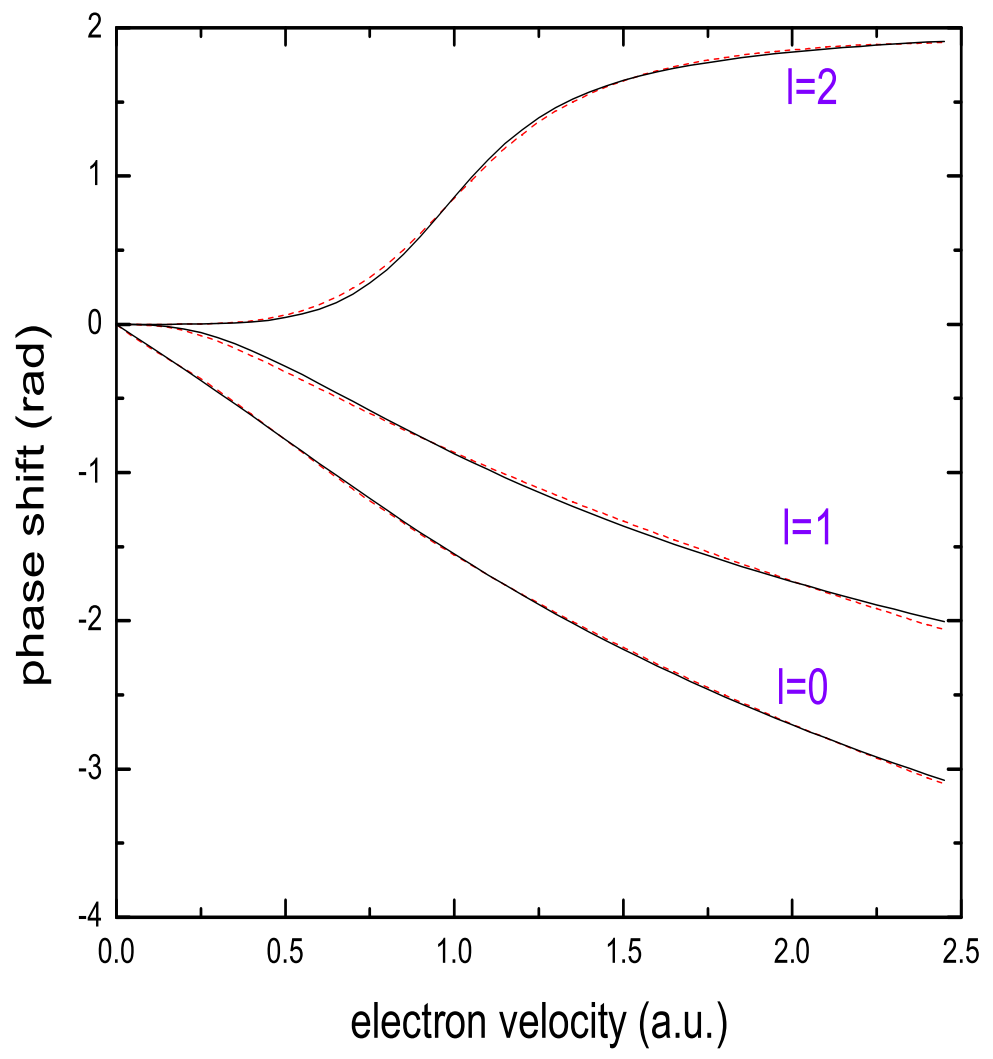

FIG. 2: Electron scattering phase shifts (modulo $\pi$ ) for Ar obtained using the static-exchange (i.e., Hartee-Fock) atomic potential (solid black lines), and the pseudopotential (3) (dashed red lines), with parameters listed in Table I.

\section{A. Ps-Kr scattering phase shifts}

Figure 3 shows the phase shifts for Ps with the orbital angular momentum $L=0,1$, and 2, scattered from Kr. Adding the van der Waals interaction (with $R_{c}=3.0$ a.u., see Sec. III C) produces relatively small positive corrections for the $s$ and $p$ waves, while for the $d$ wave the correction is relatively large. In particular, due to the van der Waals potential, the phase shift acquires a characteristic rise, $\delta_{2} \propto k^{4}$, at low Ps momenta $k$ [see Eq. (B6)].

Our calculations show that for $L=0$ and 1 , the nonlocal effects related to the second term on the right-hand side of Eq. (10) are small, but for $L=2$ they are significant. This can be seen from Fig. 4, which compares the full (nonlocal) $d$-wave phase shift with those obtained using two choices of the local potential, namely, $v_{\text {loc }}(r)=v_{1}(r)$ and $v_{\text {loc }}(r)=v_{2}(r)$, where $v_{l}(r)$ is defined by Eq. (7). The choice of $v_{\text {loc }}=v_{2}$ leads to a shape resonance due to the combination of the van der Waals interaction and the centrifugal barrier. The resonance 


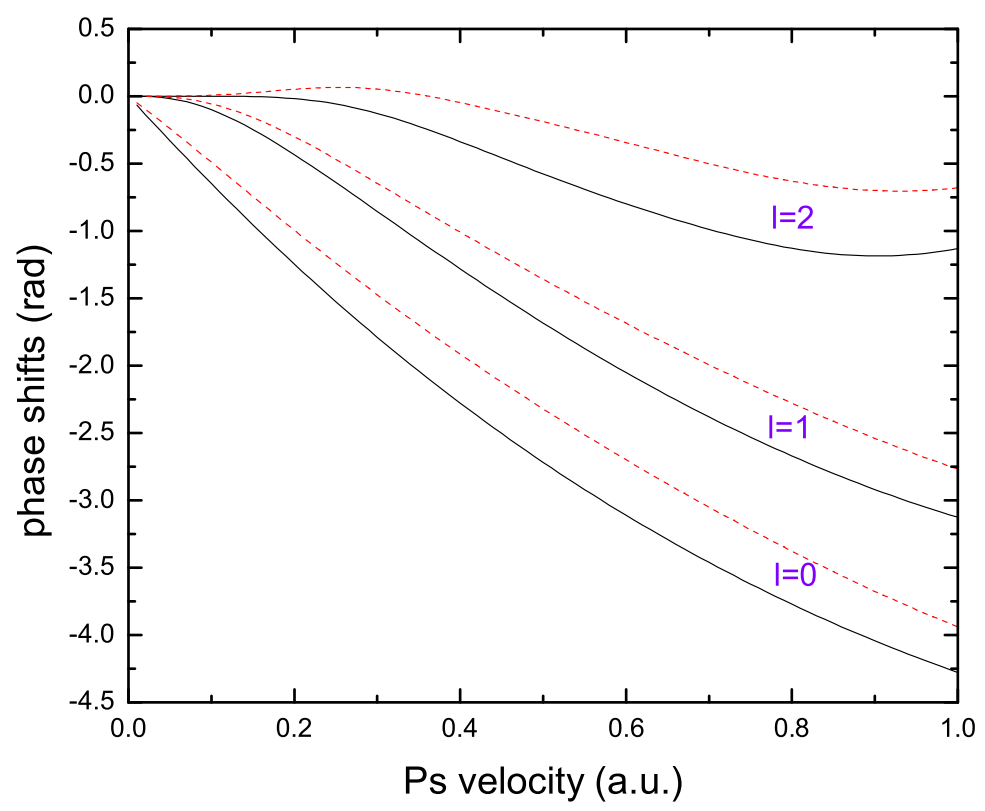

FIG. 3: (Color online) Ps-Kr scattering phase shifts for $L=0,1$ and 2. For each partial wave, the lower curve (solid black) is for the static pseudopotential, and the upper curve (dashed red) includes the effect of the van der Waals interaction with $C_{6}=152$ a.u. and $R_{c}=3.0$ a.u.

becomes very pronounced at smaller $R_{c}$. However, for $v_{\text {loc }}=v_{1}$, the resonance is not visible, and the result obtained with the full potential (i.e., including the local and nonlocal terms) confirms that the nonlocal effects suppress the resonance behavior of the Ps $d$ wave. This suppression leads to a better agreement with the static-exchange calculations of Blackwood et al. [5] (see below). A trace of this resonance is a weak minimum in the $L=2$ partial cross section (see Sec. III C below).

Analysis of the Ps $s$-wave phase shift at low momenta $\left(\delta_{0} \simeq-A k\right)$ yields the value of the scattering length $A$. In the static approximation we find $A=3.32$ a.u., and when the van der Waals interaction is included we obtain $A=2.35$ a.u. (for $R_{c}=3.0$ ), or $A=2.50$ a.u. (for $R_{c}=3.5$ ). These values can be compared with those of Mitroy and Bromley [15], i.e., $A=3.18$ a.u. in the static approximation, and $A=1.98$ a.u. with the van der Waals interaction included. Note that because of the uncertainty in the van der Waals interaction effect in Ref. [15], the corresponding scattering length varies between 1.22 and 2.26 a.u., with $A=1.98$ a.u. being their best prediction. 


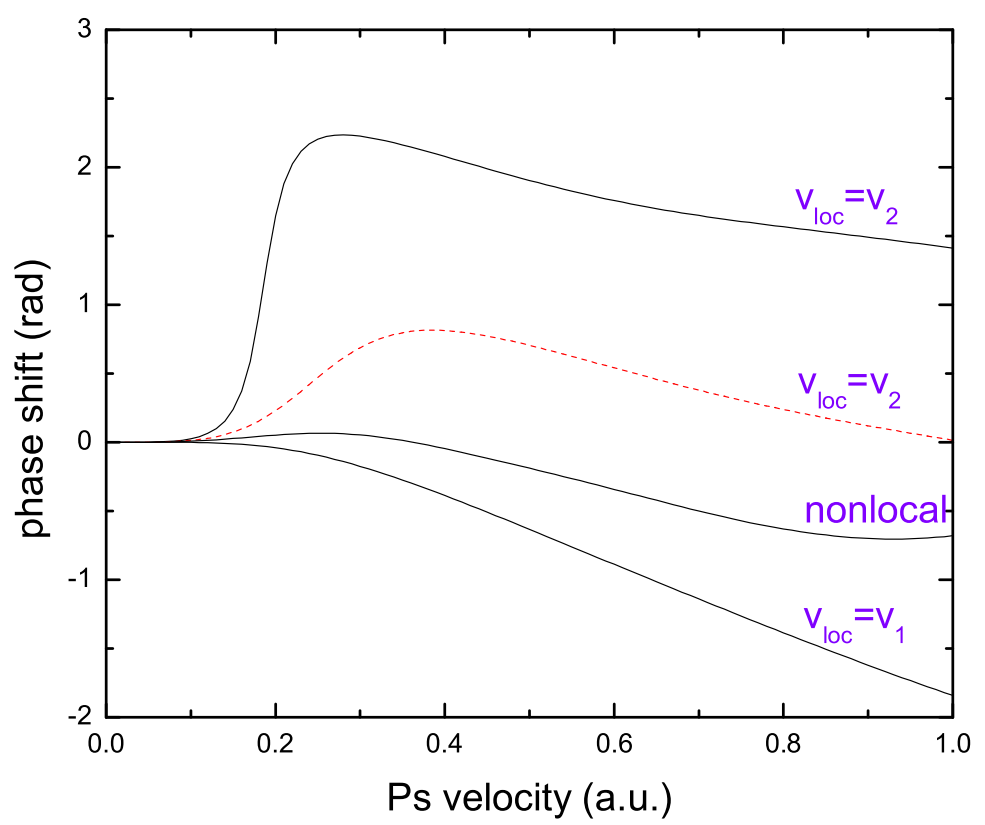

FIG. 4: (Color online) Ps-Kr scattering phase shifts for $L=2$. Shown are the results obtained with the full pseudopotential (i.e., with nonlocal effects) and its local approximations $v_{\text {loc }}(r)=v_{l}(r)$ for $l=1$ and 2. The van der Waals interaction is included in all cases. Solid black lines: $R_{c}=3.0$ a.u., red dashed line: $R_{c}=3.5$ a.u.

\section{B. Effect of the van der Waals interaction on the scattering length}

As seen from the $s$-wave phase shift in Fig. 3, the effect of the van der Waals interaction is not very significant. It does not change the sign of the scattering length (the way atomic polarization does for the electron and positron scattering), and there is no Ramsauer-Townsend minimum in the cross section. To understand this qualitatively and semiquantitatively, consider a model potential with a hard repulsive core of radius $R_{0}$ and a van der Waals "tail",

$$
V(R)=\left\{\begin{array}{ll}
+\infty, & R<R_{0} \\
-C_{6} / R^{6}, & R>R_{0}
\end{array} .\right.
$$

Here the repulsive core mimics the exchange interaction between the electron and the atom. The $s$-wave radial Schrödinger equation for $k=0$ at $R>R_{0}$ is

$$
\frac{d^{2} f_{0}}{d R^{2}}+\frac{2 m C_{6}}{R^{6}} f_{0}(R)=0 .
$$

Its solution (up to a normalization constant) is

$$
f_{0}(R)=R^{1 / 2}\left[J_{-1 / 4}\left(x_{0}\right) J_{1 / 4}(x)-J_{1 / 4}\left(x_{0}\right) J_{-1 / 4}(x)\right]
$$


where

$$
x=\frac{\sqrt{m C_{6} / 2}}{R^{2}}, \quad x_{0}=\frac{\sqrt{m C_{6} / 2}}{R_{0}^{2}},
$$

and $J_{\nu}$ is the Bessel function. The scattering length, obtained from the asymptotic behavior of Eq. (18) at $R \rightarrow \infty, f_{0}(R) \propto R-A$, is

$$
A=\left(\frac{m C_{6}}{8}\right)^{1 / 4} \frac{\Gamma(3 / 4)}{\Gamma(5 / 4)} \frac{J_{-1 / 4}\left(x_{0}\right)}{J_{1 / 4}\left(x_{0}\right)}=R_{0}\left(1-\frac{2 x_{0}^{2}}{15}-\frac{22 x_{0}^{4}}{1575}-\frac{844 x_{0}^{6}}{482625}+\ldots\right) .
$$

Here the factor $\left(m C_{6} / 8\right)^{1 / 4} \Gamma(3 / 4) / \Gamma(5 / 4)$ is similar to the mean atom-atom scattering length determined by the long-range part of the van der Waals interaction (see Ref. [16]).

Figure 5 shows the scattering length $(20)$ as a function of $R_{0}$ for three values of the van der Waals constant, $C_{6}=104.5$, 152, and 234 a.u., corresponding to Ps-Ar, Ps-Kr, and Ps-Xe interactions. The scattering length becomes negative only for unrealistically small values of $R_{0}$. As an estimate, we can assume that $R_{0}$ equals the scattering length in the static approximation. For Kr, this gives $R_{0}=3.32$ a.u. Using this value in Eq. (20), we see that the van der Waals force reduces the scattering length to $A=2.67$ a.u., in good accord with the calculations (Sec. III A). Therefore, the effect of the van der Waals interaction is not as drastic as the effect of the polarization interaction in $e^{-}-\mathrm{Kr}$ ( or $e^{+}-\mathrm{Kr}$ ) scattering, where it makes the scattering length negative. Obviously this is due to the shorter range of the van der Waals force as compared to the polarization force.

Alternatively, one can estimate $R_{0}$ using the mean radius of the outer atomic orbital $\langle r\rangle$, e.g., as

$$
R_{0}=\gamma\langle r\rangle
$$

where $\gamma \sim 1$ is a dimensionless factor. For $\mathrm{Kr},\langle r\rangle=1.95$ a.u. [17], and in order to obtain the correct scattering length, $A=2.35$ a.u. (for $R_{c}=3.0$ ), we should choose $\gamma=1.61$, which leads to $R_{0}=3.14$ a.u., close to our previous estimate of $R_{0}=3.32$ a.u.

We can use this simple model to estimate the Ps scattering lengths for other atoms. Table II lists the values of $\langle r\rangle$ from Ref. [17] and the corresponding values of the model scattering length (20) for Ar, $\mathrm{Kr}$ and Xe, obtained using $\gamma=1.61$. Also shown are the results of the scattering calculations of this work and of Mitroy et al. [9, 15].

Although the model result for Xe looks somewhat overestimated, the model correctly predicts the trend of the scattering length $A$ to increase with the atomic number $Z$. Although the van der Waals interaction (which makes $A$ smaller) grows with $Z$, the increase of the 


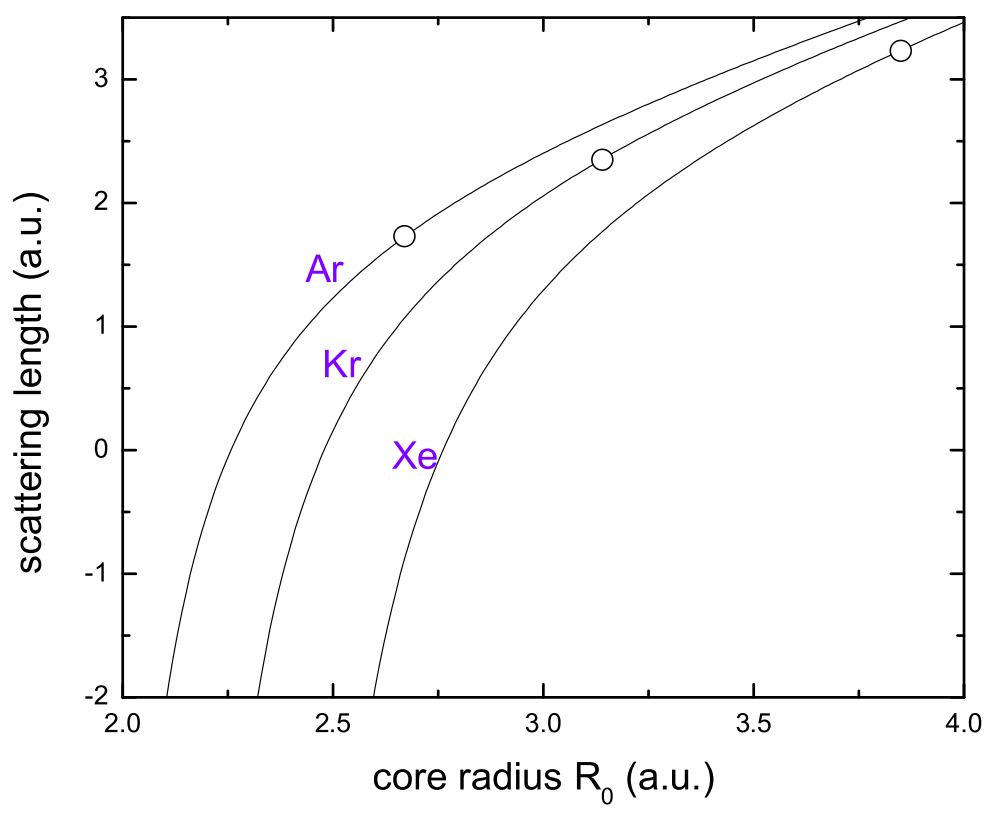

FIG. 5: Dependence of the scattering length, given by Eq. (20), for the van der Waals potential (17) on the radius of the repulsive core. The three curves correspond to $C_{6}=104.5,152$, and 234 a.u., for Ps-Ar, Ps-Kr, and Ps-Xe, respectively. Open circles show the estimates of $A$ from Table II.

TABLE II: Mean atomic radii $\langle r\rangle$, core radii $R_{0}$, and Ps-atom scattering lengths $A$ for $\mathrm{Ar}, \mathrm{Kr}$ and Xe. All values are in a.u.

\begin{tabular}{ccccccc}
\hline \hline System & $C_{6}$ & $\langle r\rangle$ & $R_{0}$ & $A^{a}$ & $A^{b}$ & $A^{c}$ \\
\hline $\mathrm{Ar}$ & 104.5 & 1.66 & 2.67 & 1.73 & $2.14-2.33$ & $1.30-1.98$ \\
$\mathrm{Kr}$ & 152 & 1.95 & 3.14 & 2.35 & $2.35-2.50$ & $1.22-2.26$ \\
$\mathrm{Xe}$ & 234 & 2.39 & 3.85 & 3.23 & - & $1.50-2.60$ \\
\hline \hline
\end{tabular}

${ }^{a}$ Scattering length from Eq. (20), obtained using Eq. (21) with $\gamma=1.61$.

${ }^{b}$ Present scattering calculations.

${ }^{c}$ Values obtained by Mitroy et al. $[9,15]$.

atomic radius, i.e., the effect of the static repulsion, is stronger. [This can be seen from the expression for $x_{0}$, given by Eq. (19), which is proportional to $C_{6}^{1 / 2}$ but inversely proportional to $R_{0}^{2}$.] This is opposite to what is observed in electron-atom scattering where the effect of the polarization attraction takes over the effect of the core radius, and the scattering length decreases with the growth of $Z$. (The analog of the parameter $x_{0}$ in this case is proportional 
to $\sqrt{\alpha} / R_{0}$, see Ref. [16].)

These observations have an important consequence for the comparison of $e^{-}-A$ scattering with Ps- $A$ scattering. The observed similarities [1] at energies above the Ps ionization threshold are explained in terms of the impulse approximation [4]. On the other hand, in the region below the ionization threshold, where the impulse approximation fails, no similarity exists. In this energy range scattering is controlled by different long-range interactions, the strong polarization interaction for the electrons, and the relatively weak van der Waals interaction in the case of Ps.

\section{Ps-Kr scattering cross section}

Figure 6 shows the elastic Ps-Kr cross sections in the velocity range from threshold to 2 a.u. Higher partial waves $(L>4)$, up to $L=10$, were included by solving the radial equation in the local approximation with inclusion of the van der Waals interaction. The van der Waals interaction was included with two choices of the cut-off parameter: $R_{c}=3.0$ and 3.5 a.u. The figure also shows the cross section obtained by Blackwood et al. [5] using the static-exchange approximation, i.e., without inclusion of virtual excitations in the target or projectile. These results can be compared with our static calculations. Figure 6 shows that the two theories are very close at low velocities, but the present static-field cross section decreases faster with the increasing velocity, compared to that of Blackwood et al. However, after inclusion of the van der Waals interaction, our cross section increases significantly at $v>0.6$ a.u., and merges with the result of the impulse approximation for $R_{c}=3.0$ a.u. We therefore choose this value of $R_{c}$ for comparison with the experiment (see below).

At low velocities (below the Ps ionization threshold, $v=0.5$ a.u.), the van der Waals interaction leads to a significant reduction of the elastic cross section. This effect is due to the decrease in the scattering length and a general increase of the low-l phase shifts (i.e., decrease in absolute magnitude), as seen in Fig. 3. The van der Waals interaction also results in two features in the cross section: a local maximum at $v=0.22$ a.u. and a local minimum at $v=0.67$ a.u. (for $R_{c}=3.0$ a.u.). The former is due to the $s$-wave contribution and is caused by the long-range attractive interaction. To understand this, it is useful to discuss a similar effect in the low-energy electron-atom scattering which is controlled by the polarization interaction. According to the modified effective-range theory of O'Malley et al. 


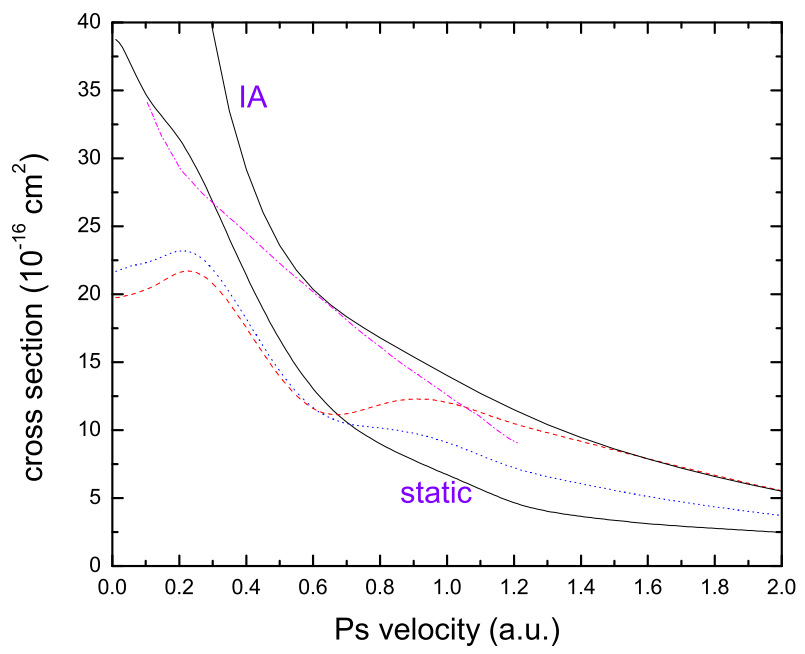

FIG. 6: (Color online) Ps-Kr elastic scattering cross sections. Solid black curve "IA" is the result of the impulse approximation [4]; solid black curve "static" is the present static-field calculation (i.e., $C_{6}=0$ ); dashed red curve is the present calculation with the static and van der Waals interaction ( $C_{6}=152$ a.u., $R_{c}=3.0$ a.u.); dotted blue curve is the same for $R_{c}=3.5$ a.u.; dot-dashed magenta curve is the static-exchange calculations of Blackwood et al. [5].

[18], at low energies the $s$-wave phase shift behaves as

$$
\tan \delta_{0}=-A k-\pi k^{2} \alpha / 3+O\left(k^{3} \ln k\right)
$$

where $\alpha$ is the atomic polarizability. Here the characteristic quadratic part of the polarization contribution to the phase shift [second term in Eq. (22)] is negative (although the total contribution of the attractive polarization potential is positive). As a result, for a negative scattering length $A$, the phase shift passes through zero at small $k>0$, leading to the Ramsauer-Townsend effect. In contrast, for $A>0$ the phase shift decreases faster than linear, which gives rise to a maximum in the partial cross section. For example, a maximum is observed in electron scattering from $\mathrm{Ne}$ [19], for which the scattering length is small and positive. (Note that although the maximum in the total cross section for Ne is observed at about $E=25 \mathrm{eV}$, the $s$-wave contribution peaks at $E=6.7 \mathrm{eV}$, still quite a large energy compared to the position of a typical Ramsauer-Townsend minimum.) One could call this phenomenon the "anti-Ramsauer" effect, although we are not aware of the use of such term in the literature. 
A similar situation occurs in Ps-atom scattering, although now the additional contribution to the phase shift comes from the van der Waals interaction. As shown in Appendix B, the modified effective-range expansion of the $s$-wave scattering phase shift reads as

$$
\tan \delta_{0}=-A k-B k^{3}+2 m C_{6} \pi k^{4} / 15+O\left(k^{5}\right),
$$

where $B=\frac{1}{2} r_{0} A^{2}$, and $r_{0}$ is the effective range [20]. The coefficient $B$ depends on both the short-range and the long-range (van der Waals) interactions. If $B>0$, the major correction to the $-A k$ behavior is negative, since the $k^{4}$ term is relatively small at low energies. Our calculations show that this is indeed the case, although the expansion (23) is valid only at very low energies. Hence, a weak anti-Ramsauer effect is observed. Naturally, the effect is small compared to that observed in electron-atom scattering because of the relative weakness of the van der Waals interaction compared to the polarization interaction.

The minimum at $v=0.67$ a.u. observed in Fig. 6 for $R_{c}=3.0$ a.u. is due to the $d$-wave shape resonance, which is quite pronounced in the $e^{-}-\mathrm{Kr}$ scattering [21]. However, in Ps-Kr scattering, this resonance is suppressed, as discussed in Sec. III A and seen in Figs. 3 and 4. Due to a strong background contribution, this resonance appears as a window.

To compare with the experimental Ps-atom total scattering cross section, the Ps ionization cross section should be added to the elastic cross section. Indeed, Ps ionization contributes significantly at velocities $v>0.5$ a.u. As in the impulse-approximation calculations [4], the ionization cross sections are taken from Ref. [22]. Figure 7 shows the elastic and total cross section computed in the present work together with the results of the impulse approximation and experiment. Although the experimental data [1] are not available at low velocities, the data point at $v=0.63$ a.u. indicates that the cross section should slightly decrease towards lower velocities. This trend is confirmed by our results. The peaking of the experimental cross section at $v \approx 0.9$ a.u. is also in agreement with our results. In addition, our calculation predicts a weak local maximum at $v=0.23$ a.u. and a local minimum at $v=0.56$ a.u. Both of these predictions call for experimental verification.

\section{Ps-Ar scattering}

Figure 8 shows the $s-, p$ - and $d$-wave phase shifts for Ps-Ar scattering. All of the main features here are the same as in the Ps-Kr scattering. However, $\tan \delta_{0}$ follows the linear 


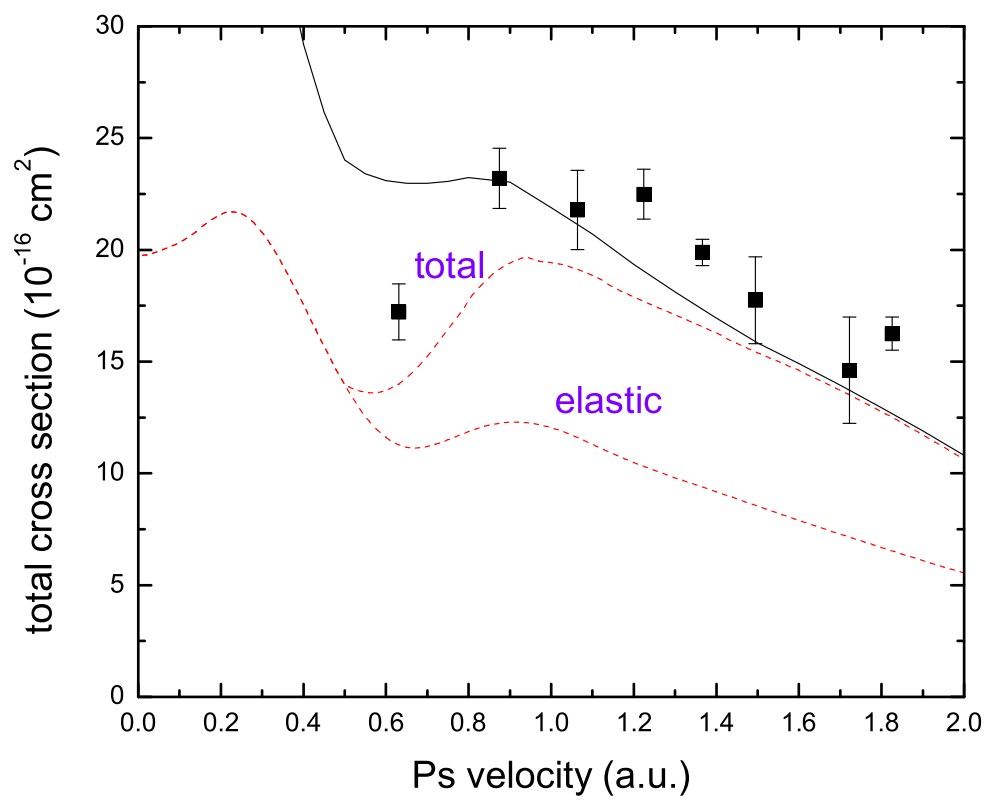

FIG. 7: (Color online) Ps-Kr total scattering cross sections. Solid black curve is the total cross section in the impulse approximation [4]; dashed red curves present elastic and total cross sections obtained with $C_{6}=152$ a.u., $R_{c}=3.0$ a.u. Solid squares are experimental data from Ref. [1].

- Ak behavior much more closely. This results in a decrease of the $s$-wave cross section, according to

$$
\sigma_{0} \simeq 4 \pi \frac{A^{2}}{1+A\left(A-r_{0}\right) k^{2}},
$$

where $r_{0}$ is, in fact, quite small. At the same time the $p$-wave contribution increases rather sharply from threshold, leading to a local maximum in the total cross section at $v=0.22$ a.u. in the static approximation $\left(C_{6}=0\right)$, and an even more pronounced local maximum at $v=0.26$ a.u. when the van der Waals interaction is included. The total elastic cross sections are shown in Fig. 9.

The local maximum in the total cross section is controlled by the local part of the Ps-Ar pseudopotential for $L=0$ and $L=1$. Since this potential is very sensitive to the partial cancellation of the attractive part due to the $e^{-}$-Ar interaction and the repulsive part due to the $e^{+}-\mathrm{Ar}$ interaction, the position and the magnitude of the maximum is subject to uncertainties. It is possible that effects not included in the present calculations, e.g., shortrange correlations, can change the position and shape of the maximum or even eliminate it completely. We note that the close-coupling calculations of Blackwood et al. [5], which allow for the virtual excitations of the Ps, but keep the target atom frozen, do not exhibit 


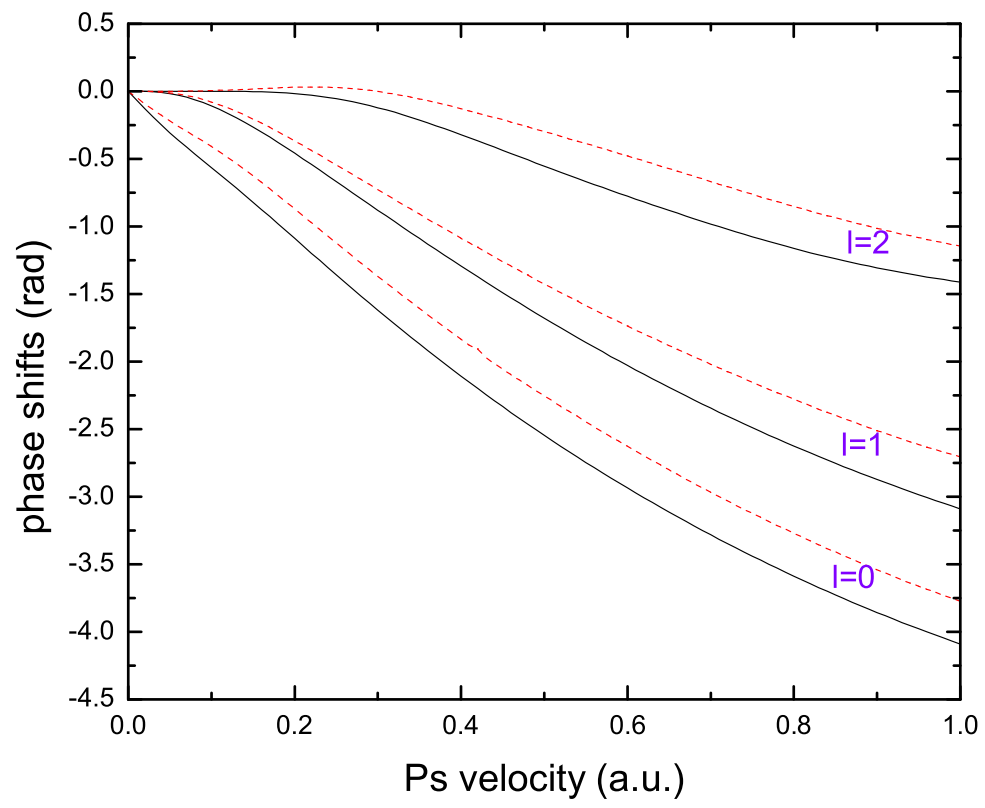

FIG. 8: (Color online) Ps-Ar scattering phase shifts. For each $L$, the lower curve (solid black) was calculated using the static-field pseudopotential, while the upper (dashed red) also includes the effect of the van der Waals interaction with $C_{6}=104.52$ a.u. and $R_{c}=3.0$ a.u.

a low-energy maximum at all. However, they do show a local maximum at $v=1.1$ a.u., which in our calculation is due to the $d$-wave contribution. Overall, Fig. 9 shows that there is a reasonable agreement with calculations [5] for $v=0.4-1.2$ a.u.. One can also see that the effect of the van der Waals potential for Ar is smaller than it is for Kr (Fig. 6), and the cross section is not as sensitive to the choice of $R_{c}$ (which can be related to the smaller $C_{6}$ value for $\mathrm{Ar}$ ). For a more detailed comparison between the present calculation and that of Blackwood et al. [5], information on partial cross sections would be needed.

The Ps-Ar scattering length in the static approximation is $A=3.19$ a.u., whereas the value calculated by Mitroy and Ivanov [9] is 2.85 a.u. After adding the van der Waals interaction we obtain $A=2.33$ a.u. for $R_{c}=3.0$ a.u., and $A=2.14$ a.u. for $R_{c}=2.5$ a.u. The corresponding value obtained by Mitroy and Ivanov varies between 1.30 and 1.98 a.u. Comparing with the Ps scattering lengths obtained for Kr (Sec. III A), we see that the values for both atoms are quite close. While the Ar-Kr system has a smaller $C_{6}$ value, the Ar atom has a smaller radius, and the two effects largely compensate for each other (see Fig. 5 and Table II).

As seen in Fig. 9, the choice of $R_{c}=3.0$ a.u. matches better with the impulse ap- 


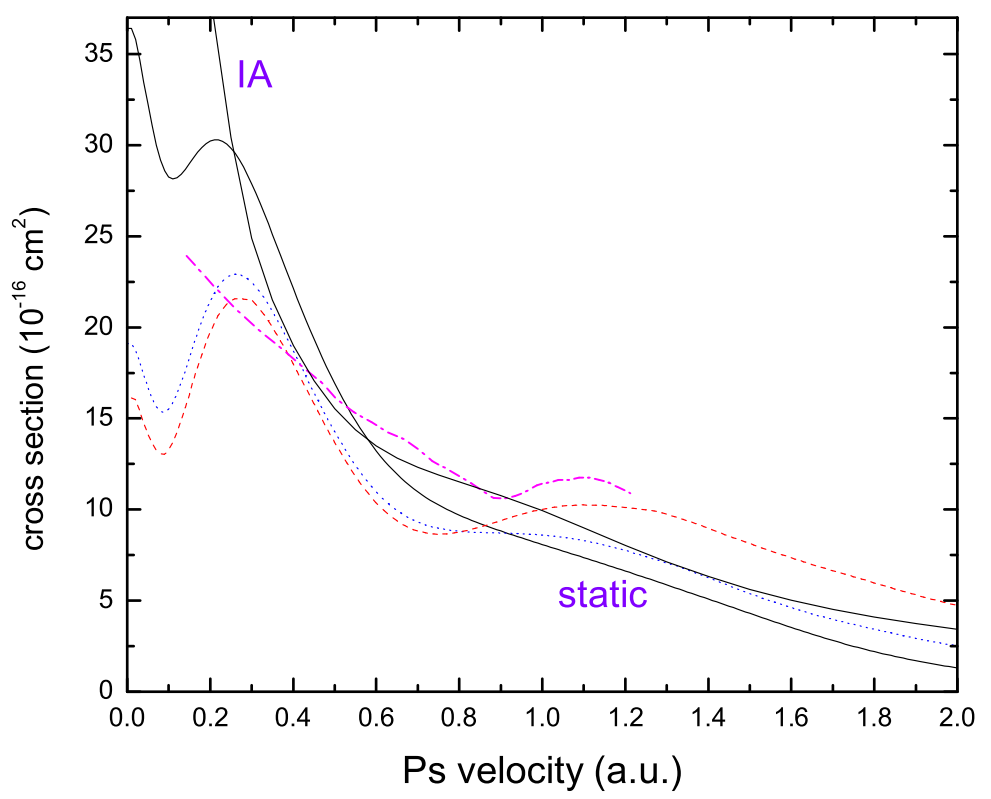

FIG. 9: (Color online) Ps-Ar elastic scattering cross sections. Solid black curve "IA" is the result of the impulse approximation [4]; solid black curve "static" is the present static calculation $\left(C_{6}=0\right)$; dashed red curve is the calculation with the static and van der Waals potential $\left(C_{6}=104.5\right.$ a.u., $R_{c}=2.5$ a.u.); dotted blue curve is the same for $R_{c}=3.0$ a.u.; dot-dashed magenta curve is the close-coupling calculations of Blackwood et al. [5].

proximation results at higher velocity. However, the smaller value of $R_{c}=2.5$ a.u. leads to a better agreement with the experiment. Figure 10 shows the total cross sections for Ps-Ar scattering obtained for each of these cut-off radii. Whereas both theoretical curves describe well the overall behavior of the measured cross sections [23] at $v>0.5$ a.u., the cross section obtained with $R_{c}=2.5$ a.u. agrees better with the measured absolute values. This value also appears to be more physical, as it is smaller than the optimal cut-off radius of $R_{c}=3.0$ a.u. found for $\mathrm{Kr}$.

\section{CONCLUSION}

The pseudopotential model developed in the present paper describes Ps scattering from noble-gas atoms (Ar and $\mathrm{Kr}$ ) at energies below the ionization threshold and matches the impulse-approximation results above the ionization threshold. Although experimental data are not available in the low-energy region, our results describe well the trend seen in the 


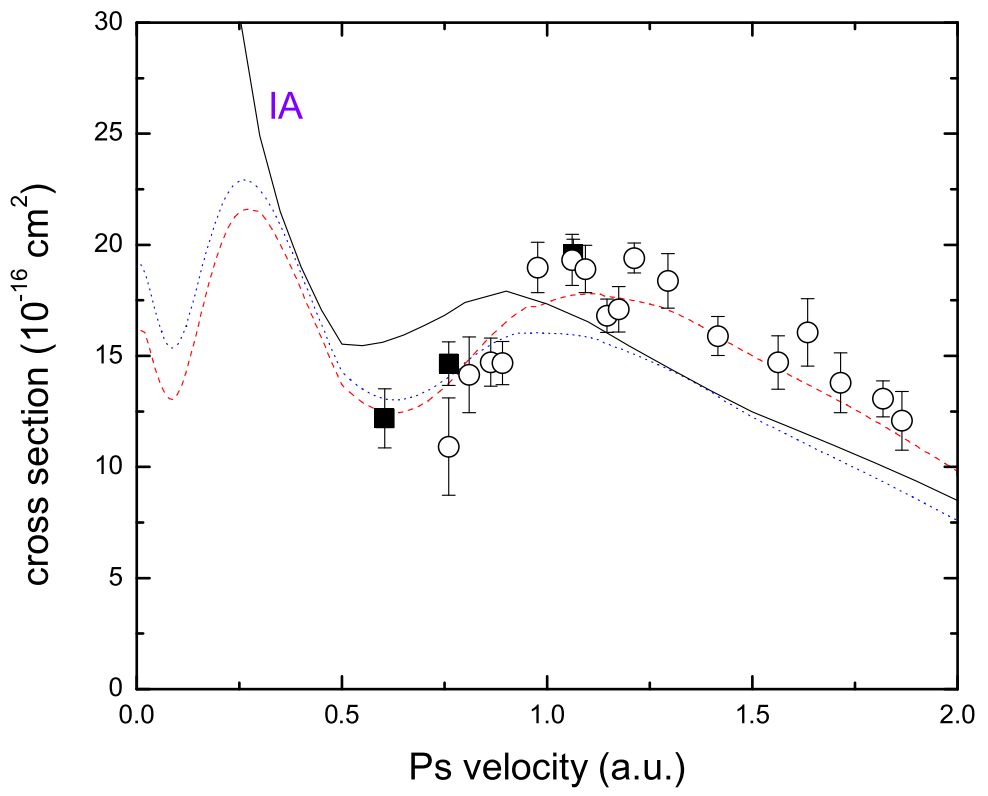

FIG. 10: (Color online) Ps-Ar total scattering cross sections. Solid black curve "IA" is the result of the impulse approximation [4]; dashed red curve is the present elastic cross section obtained with $C_{6}=104.5$ a.u., $R_{c}=2.5$ a.u., augmented by the ionization cross sections of Starrett et al. [22]; dotted blue curve is the same for $R_{c}=3.0$ a.u. Open circles: experiment [23]; solid squares: experiment [1].

experimental cross sections to drop with decreasing velocity below $v \approx 1$ a.u. In addition, our calculations predict zero-energy cross sections (or scattering lengths) which are in accord with stochastic variational calculations $[9,15]$.

Analysis of the scattering phase shifts shows that the static Ps-atom interaction is repulsive. This repulsion arises from the electron Pauli exclusion from closed-shell atoms (while the pure electrostatic interaction is zero for the truly neutral Ps atom). The phase shifts also indicate that the role of correlations represented by the van der Waals interaction at low energies, is relatively small.

Because of the relative weakness of the van der Waals interaction compared to the polarization interaction in electron-atom scattering, the scattering lengths for both Ar and $\mathrm{Kr}$ are positive, and the Ramsauer-Townsend minimum is not observed for these targets. The overall picture of Ps- $A$ scattering is quite different from the $e^{-}-A$ scattering in the low-energy region. This is in stark contrast to the intermediate energy range from the Ps ionization threshold up to $v \sim 2$ a.u. Here the Ps- $A$ scattering is mostly controlled by the 
electron-atom exchange, which makes its cross section very similar to that for $e^{-}-A$ scattering. In the low-energy region, where the long-range interaction is important (especially for the electrons), this similarity disappears.

Although the van der Waals interaction in Ps- $A$ scattering does not produce the Ramsauer-Townsend minimum, it can lead to more subtle features in the cross sections, such as low-energy maxima. However these features are subject to uncertainties because of a delicate balance between the repulsive and attractive components of the Ps- $A$ interaction. They can also be affected by other effects such as short-range correlations, which are not included explicitly in the present calculation. The uncertainty can be resolved by performing accurate measurements of Ps- $A$ scattering at low energies, and by new fully correlated calculations for this interesting and challenging system.

\section{Acknowledgments}

The authors are grateful to G. Laricchia for stimulating discussions.

\section{Appendix A: Expansion of the Ps ground state density}

We start with a known expression for the free-particle Green's function (see, e.g., [24]),

$$
\frac{e^{i k r_{12}}}{r_{12}}=i k \sum_{l=0}^{\infty}(2 l+1) h_{l}^{(1)}\left(x_{>}\right) j_{l}\left(x_{<}\right) P_{l}\left(\cos \theta_{12}\right)
$$

where $x_{>}=k r_{>}, x_{<}=k r_{<}$, and $j_{l}, h_{l}^{(1)}$ are the spherical Bessel and Hankel functions.

To switch to the decaying exponent, we make a substitution $k=i \kappa$ and arrive at the following expansion

$$
e^{-\kappa r_{12}}=\sum_{l=0}^{\infty}(2 l+1) F_{l}\left(r_{1}, r_{2}\right) P_{l}\left(\cos \theta_{12}\right)
$$

where

$$
F_{l}\left(r_{1}, r_{2}\right)=\frac{d}{d \kappa}\left[\kappa h_{l}^{(1)}\left(i \kappa r_{>}\right) j_{l}\left(i \kappa r_{<}\right)\right]
$$

It is convenient now to introduce the following real functions:

$$
\hat{h_{l}}(x)=-i^{l} h_{l}^{(1)}(i x), \quad \hat{j}_{l}(x)=i^{l} j_{l}(i x)
$$


which are related to the modified Bessel function $K_{l+1 / 2}(x)$ and $I_{l+1 / 2}(x)$. Explicit expressions for the first few of these functions are

$$
\begin{gathered}
\hat{h}_{0}(x)=\frac{e^{-x}}{x}, \quad \hat{h}_{1}(x)=\frac{e^{-x}}{x}\left(1+\frac{1}{x}\right), \quad \hat{h}_{2}(x)=\frac{e^{-x}}{x}\left(1+\frac{3}{x}+\frac{3}{x^{2}}\right) \\
\hat{j}_{0}(x)=\frac{\sinh x}{x}, \quad \hat{j}_{1}(x)=\frac{1}{x}\left(\frac{\sinh x}{x}-\cosh x\right), \quad \hat{j}_{2}(x)=\frac{\sinh x}{x}\left(1+\frac{3}{x^{2}}\right)-\frac{3 \cosh x}{x^{2}} .
\end{gathered}
$$

The recurrence relations for these functions are

$$
\begin{aligned}
& z_{l+1}(x)=\frac{2 l+1}{x} z_{l}(x)+z_{l-1}(x), \\
& \frac{d z_{l}(x)}{d x}=-\frac{l+1}{x} z_{l}(x)-z_{l-1}(x),
\end{aligned}
$$

where $z_{l}$ stands for either $\hat{j}_{l}$ or $\hat{h}_{l}$. Using these, we obtain

$$
F_{0}\left(x_{1}, x_{2}\right)=-\hat{h}_{0}\left(x_{>}\right) \hat{j}_{0}\left(x_{<}\right)+x_{>} \hat{h}_{1}\left(x_{>}\right) \hat{j}_{0}\left(x_{<}\right)+x_{<} \hat{h}_{0}\left(x_{>}\right) \hat{j}_{1}\left(x_{<}\right)
$$

and

$$
F_{l}\left(x_{1}, x_{2}\right)=(-1)^{l}\left[(2 l+1) \hat{h}_{l}\left(x_{>}\right) \hat{j}_{l}\left(x_{<}\right)+x_{>} \hat{h}_{l-1}\left(x_{>}\right) \hat{j}_{l}\left(x_{<}\right)+x_{<} \hat{h}_{l}\left(x_{>}\right) \hat{j}_{l-1}\left(x_{<}\right)\right], \quad l \geq 1 .
$$

In practice, expansion (A1) converges well by summing up to $l_{\max }=30$ if, for high $l$, asymptotic expansions for $\hat{h}_{l}$ and $\hat{j}_{l}$ are used.

\section{Appendix B: Modified effective range expansion for the van der Waals potential}

We are interested in the behavior of the $s$-wave scattering phase shift $\delta_{0}$. According to the effective range theory for short-range potentials, it is given by the effective-range expansion $[25]$

$$
k \cot \delta_{0}=-\frac{1}{A}+\frac{1}{2} r_{0} k^{2}+O\left(k^{4}\right)
$$

where $A$ is the scattering length and $r_{0}$ is the effective range. Note that in the presence of a weakly bound state, $r_{0}>0$ [20], but generally this is not true. For small momenta and phase shifts, it is more convenient to rewrite Eq. (B1) as

$$
\tan \delta_{0}=-A k-B k^{3}+O\left(k^{5}\right)
$$

where $B=\frac{1}{2} A^{2} r_{0}$. More generally, $k \cot \delta_{0}$ in Eq. (B1) can be expanded in even powers of $k$, and $\tan \delta_{0}$ in Eq. (B2) in odd powers of $k$. 
In the presence of the long-range interaction $-C_{n} / r^{n}$ the first "anomalous" term in the expansion (B2) is proportional to $k^{n-2}$ [20]. This term can be calculated in the Born approximation according to the prescription given by Landau and Lifshitz [20]. Consider the case $n=6$ (van der Waals interaction). The corresponding correction $\Delta f$ to the scattering amplitude is

$$
\Delta f(q)=2 m C_{6} q^{3} \int_{q R_{c}}^{\infty} \frac{\sin \xi}{\xi^{5}} d \xi,
$$

where $q=2 k \sin \theta / 2$ is the momentum transfer, $\theta$ is the scattering angle, and $R_{c}$ is a cutoff radius similar to that introduced in Eq. (2). Integrating several times by parts and expanding the result in powers of $k R_{c}$ at small $k R_{c}$ gives

$$
\Delta f(q) \approx 2 m C_{6}\left(\frac{1}{3 R_{c}^{3}}-\frac{q^{2}}{6 R_{c}}+\frac{\pi q^{3}}{48}\right) .
$$

Expanding this amplitude in partial waves, we obtain for the correction to the $s$-wave phase shift:

$$
\Delta \delta_{0}=c_{1} k+c_{2} k^{3}+\frac{2 \pi m C_{6} k^{4}}{15}
$$

where

$$
c_{1}=\frac{2 m C_{6}}{3 R_{c}^{3}}, \quad c_{2}=-\frac{2 m C_{6}}{R_{c}} .
$$

The first two terms in the Eq. (B3) expression are of the same type as those in the effectiverange expansion (B2), while the last term is "anomalous", caused by the power-law behavior of the potential. Therefore, the modified effective-range expansion can be written as

$$
\tan \delta_{0}=-A^{\prime} k-B^{\prime} k^{3}+\frac{2 \pi m C_{6} k^{4}}{15}+O\left(k^{5}\right),
$$

where we have introduced the new parameters $A^{\prime}, B^{\prime}>0$ to emphasize that they are different from those in Eq. (B2).

One might ask if this derivation is rigorous enough because of the use of the Born approximation. In fact, the expansion in Eq. (B4) can be derived from a more rigorous modified effective-range theory [26] for the $-C_{n} / r^{n}$ potential. This theory shows that the first "anomalous" correction to the effective-range expansion of the phase shift can be obtained from the analytical continuation of the integral [26],

$$
\Delta \delta_{L}=\pi m C_{n} k^{n-2} \int_{0}^{\infty} \frac{\left[J_{L+1 / 2}(x)\right]^{2}}{x^{n-1}} d x
$$


which converges for $L>(n-3) / 2$, to any physical value of $L$, e.g., $L=0$ for the $s$-wave scattering. [In (B5), $J_{\nu}$ is the Bessel function.] For $n=6$, one obtains (see also Refs. [20, 27])

$$
\Delta \delta_{L}=\frac{6 \pi m C k^{4}}{(2 L+5)(2 L+3)(2 L+1)(2 L-1)(2 L-3)},
$$

which means that the lowest order correction in the effective range expansion for $L=0$ is

$$
\Delta \delta_{0}=\frac{2 \pi m C_{6} k^{4}}{15}
$$

in agreement with Eq. (B3).

For a short-range potential, the low-energy behavior of the higher partial wave phase shifts is $\delta_{L} \propto k^{2 L+1}$. This means that for $2 L+1>4$, i.e., $L \geq 2$, the "anomalous" correction (B6) is, in fact, the leading term in the low- $k$ expansion. This explains the behavior of the $d$-wave phase shifts seen in Figs. 3 and 8 when the van der Waals interaction is included.

[1] S. J. Brawley, S. Armitage, J. Beale, D. E. Leslie, A. I. Williams, and G. Laricchia, Science 330, 789 (2010).

[2] S. J. Brawley, A. I. Williams, M. Shipman, and G. Laricchia, Phys. Rev. Lett. 105, 263401 $(2010)$

[3] S. J. Brawley, A. I. Williams, M. Shipman, and G. Laricchia, J. Phys. Conf. Series 388, 012016 (2012).

[4] I. I. Fabrikant and G. F. Gribakin, Phys. Rev. Lett. 112, 243201 (2014).

[5] J. E. Blackwood, M. T. McAlinden, and H. R. J. Walters, J. Phys. B 35, 2661 (2002); 36, 797 (2003).

[6] C. P. Campbell, M. T. McAlinden, F. G. R. S. MacDonald. and H. R. J. Walters, Phys. Rev. Lett. 80, 5097 (1998).

[7] J. E. Blackwood, M. T. McAlinden, and H. R. J. Walters, Phys. Rev. A 65, 032517 (2002).

[8] J. Pascale, Phys. Rev. A 28, 632 (1983).

[9] J. Mitroy and I. A. Ivanov, Phys. Rev. A 65, 012509 (2001).

[10] Using $\mathbf{r}_{e}=\mathbf{R}+\boldsymbol{\rho} / 2$ and $\mathbf{r}_{p}=\mathbf{R}-\boldsymbol{\rho} / 2$, where $\mathbf{R}$ and $\boldsymbol{\rho}$ are the Ps center-of-mass and relative electron-positron position vectors, expanding $V_{\text {pol }}$ from Eq. (1) to the second order in $\rho / R$, and averaging over the Ps ground state, one obtains $V_{W}(R) \simeq-C_{6} / R^{6}$ with $C_{6}=\alpha\left\langle\rho^{2}\right\rangle$, 
where $\left\langle\rho^{2}\right\rangle=12$ a.u. is the average over the Ps ground state. This is a known estimate for the van der Waals constant valid when the characteristic excitation energy of one system (Ps) is much smaller than that of the other (e.g., a noble-gas atom); see, e.g., E. E. Nikitin and B. M. Smirnov, Atomic and Molecular Processes (Nauka, Moscow, 1988).

[11] F. London, Trans. Faraday Soc. 33, 8 (1937); we use $C_{6}=3 \alpha_{1} \alpha_{2} I_{1} I_{2} /\left[2\left(I_{1}+I_{2}\right)\right]$, where $\alpha_{1,2}$ and $I_{1,2}$ are the dipole polarizabilities and ionization potentials of the two species.

[12] J. Mitroy and M. W. J. Bromley, Phys. Rev. A 68, 035201 (2003).

[13] V. A. Dzuba, V. V. Flambaum, G. F. Gribakin and W. A. King, J. Phys. B 29, 3151 (1996).

[14] The phase shifts were calculated using the codes based on those described in M. Ya. Amusia and L. V. Chernysheva, Computation of Atomic Processes. A Handbook for the ATOM Programs (IOP Publishing, Bristol, 1997).

[15] J. Mitroy and M. W. J. Bromley, Phys. Rev. A 67, 034502 (2003).

[16] G. F. Gribakin and V. V. Flambaum, Phys. Rev. A 48, 546-53 (1993).

[17] A. A. Radtzig and B. M. Smirnov, Reference Data on Atoms, Molecules, and Ions (SpringerVerlag, Berlin, 1985).

[18] T. F. O’Malley, L. Spruch, and L. Rosenberg, J. Math. Phys. 2, 491 (1961).

[19] R. P. McEachran and A. D. Stauffer, J. Phys. B 16, 4023 (1983).

[20] L. D. Landau and E. M. Lifshitz, Quantum Mechanics, 3rd ed. (Butterworth-Heinemann, Amsterdam, 1977), Chap. XVII.

[21] R. P. McEachran and A. D. Stauffer, J. Phys. B 17, 2507 (1984).

[22] C. Starrett, M. T. McAlinden, and H. R. J. Walters, Phys. Rev. A 72, 012508 (2005).

[23] A. J. Garner, A. Özen, and G. Laricchia, J. Phys. B 33, 1149 (2000).

[24] D. A. Varshalovich, A. N. Moskalev and V. K. Khersonskii, Quantum Theory of Angular Momentum (World Scientific, Singapore, 1988).

[25] H. A. Bethe, Phys. Rev. 76, 38 (1949).

[26] I. I. Fabrikant, J. Phys. B 12, 3599 (1979).

[27] P. S. Ganas, Phys. Rev. A 5, 1684 (1972). 